Notre Dame Journal of Formal Logic Volume 37, Number 3, Summer 1996

\title{
On Elementary Equivalence for Equality-free Logic
}

\author{
E. CASANOVAS, P. DELLUNDE, and R. JANSANA
}

\begin{abstract}
This paper is a contribution to the study of equality-free logic, that is, first-order logic without equality. We mainly devote ourselves to the study of algebraic characterizations of its relation of elementary equivalence by providing some Keisler-Shelah type ultrapower theorems and an Ehrenfeucht-Fraïssé type theorem. We also give characterizations of elementary classes in equalityfree logic. As a by-product we characterize the sentences that are logically equivalent to an equality-free one.
\end{abstract}

1 Introduction In first-order logic it is common to employ one symbol for the equality relation. Equality is considered a logical notion, with a fixed meaning. This was not the case when the first investigations in mathematical logic took place, but this practice has been strongly supported by successful applications to mathematical theories. Thus, the general study of first-order logic without equality, or equality-free logic, as we prefer to call it, has been neglected in favor of the more powerful version with equality. Recently some interest in fragments of equality-free logic has arisen in the frame of algebraic logic (see Blok and Pigozzi [5] and Bloom [2]). We think that a model-theoretic study of equality-free logic is worthwhile by itself and we hope that, by means of contrast with the well-known results for first-order logic, this study will contribute to the understanding of the role of equality in mathematical theories and structures. As an easy example of this comparison consider the fact that every satisfiable set of equality-free sentences has an infinite model.

Let $L$ be a similarity type. The set of equality-free formulas of $L$, that is, the set of all first-order formulas of $L$ not containing the equality symbol, is denoted by $L^{-}$. Given two $L$-structures $\mathfrak{A}, \mathfrak{B}$ with $\mathfrak{A} \equiv^{-} \mathfrak{B}$ we mean that $\mathfrak{A}$ and $\mathfrak{B}$ satisfy exactly the same sentences of $L^{-}$. We devote this paper to the study of algebraic characterizations of the relation $\equiv^{-}$and of elementary classes in the sense of $L^{-}$.

In Section 2 the Leibniz congruence of a structure $\mathfrak{A}, \Omega(\mathfrak{A})$, is introduced. It is the greatest congruence on $\mathfrak{A}$. This notion was already considered a long time ago Received July 17, 1995; revised May 28, 1996 
(see, for example, Monk [9]) but its name and its present interest mainly come from Blok and Pigozzi [4]. The quotient $\mathfrak{A}^{*}=\mathfrak{A} / \Omega(\mathfrak{A})$ is called the reduction of $\mathfrak{A}$. Following Blok and Pigozzi $[3]$ we say that two structures $\mathfrak{A}, \mathfrak{B}$ are relatives and we write $\mathfrak{A} \sim \mathfrak{B}$ if they have isomorphic reductions. It turns out that $\sim$ is a weak notion of isomorphism which is appropriate for equality-free logic. In Proposition 2.6 we give several conditions equivalent to $\mathfrak{A} \sim \mathfrak{B}$ and in Theorem 2.8 we prove that for any structures $\mathfrak{A}, \mathfrak{B}, \mathfrak{A} \equiv^{-} \mathfrak{B}$ is equivalent to the existence of elementary extensions $\mathfrak{C}, \mathfrak{D}$ of $\mathfrak{A}$ and $\mathfrak{B}$ such that $\mathfrak{C} \sim \mathfrak{D}$. From this we obtain in Theorem 2.9 a first algebraic characterization of elementary classes in $L^{-}$. It is well-known (see [9]) that an equality-free sentence is preserved under strict homomorphic images and pre-images. Here we prove the converse in Corollary 2.10: a first-order sentence which is preserved under strict homomorphic images and pre-images is equivalent to an equalityfree sentence.

In Section 3 we generalize the Keisler-Shelah theorem on isomorphic ultrapowers to the equality-free case (Theorems 3.1 and 3.3), What we obtain is that $\mathfrak{A} \equiv^{-} \mathfrak{B}$ is equivalent to the existence of an ultrafilter $U$ such that $\mathfrak{A}^{U} \sim \mathfrak{B}^{U}$. Then we restrict our attention to relational similarity types and we get stronger results (see Theorem 3.8 and Corollary 3.9. We use these theorems to obtain new algebraic characterizations of elementary classes in equality-free logic (Theorems 3.4 and 3.10. In Section 4 back and forth systems for equality-free logic are introduced. We obtain an analogue of the Ehrenfeucht-Fraïssé theorem (Proposition 4.5) and we state without proofs the basic facts about infinite back and forth.

Our notation and terminology is standard. If $\left(\mathfrak{A}_{i}\right)_{i \in I}$ is a family of structures and $U$ is an ultrafilter over $I, \prod_{i \in I} \mathfrak{A}_{i}$ is the direct product of the family and $\prod_{i \in I} \mathfrak{A}_{i} / U$ is the ultraproduct modulo $U$. We denote by $\mathfrak{A}^{I}$ the direct power of $\mathfrak{A}$ and by $\mathfrak{A}^{U}$ the ultrapower modulo $U$. In Section 3 we consider some similar although different constructions: the ultrafilter-product $\prod_{i \in I}^{U} \mathfrak{A}_{i}$ and the ultrafilter-power $\prod^{U} \mathfrak{A}$. If $L$ is a similarity type, $L_{0}$ is the set of quantifier-free formulas of $L$ and $L_{0}^{-}$is the set of quantifier-free and equality-free formulas of $L$. We write $\mathfrak{A} \equiv_{0} \mathfrak{B}$ and $\mathfrak{A} \equiv_{0}^{-} \mathfrak{B}$ to mean that $\mathfrak{A}$ and $\mathfrak{B}$ satisfy exactly the same sentences of $L_{0}$ and $L_{0}^{-}$respectively. If $\mathfrak{A}$ is an $L$-structure and $B \subseteq A$, we denote by $L(B)$ the similarity type obtained from $L$ by adding a new constant symbol for each element of $B$ and by $\mathfrak{A}_{B}$ the natural expansion of $\mathfrak{A}$ to $L(B)$ where every new constant denotes its corresponding element.

2 Relativeness and the Leibniz congruence We begin by defining the notion of strict homomorphism. This terminology comes from Czelakowski [6. In 9 these homomorphisms are called two-way homomorphisms. They should not be confused with strong homomorphisms in the sense of Chang and Keisler [7].

Definition 2.1 If $\mathfrak{A}$ and $\mathfrak{B}$ are $L$-structures, we say that a homomorphism $h: \mathfrak{A} \rightarrow$ $\mathfrak{B}$ is strict if for every $n$-ary relation symbol $R \in L$ and for every $a_{1}, \ldots, a_{n} \in A$,

$$
\left\langle a_{1}, \ldots, a_{n}\right\rangle \in R^{\mathfrak{A}} \operatorname{iff}\left\langle h\left(a_{1}\right), \ldots, h\left(a_{n}\right)\right\rangle \in R^{\mathfrak{B}} .
$$

Lemma 2.2 Let $\mathfrak{A}$ and $\mathfrak{B}$ be L-structures and h a homomorphism from $\mathfrak{A}$ onto $\mathfrak{B}$. Then the following are equivalent. 
(i) $h$ is a strict homomorphism.

(ii) $(\mathfrak{A}, a)_{a \in A} \equiv_{0}^{-}(\mathfrak{B}, h(a))_{a \in A}$.

(iii) $(\mathfrak{A}, a)_{a \in A} \equiv^{-}(\mathfrak{B}, h(a))_{a \in A}$.

Proof: Left to the reader.

In particular, if there is a strict homomorphism from $\mathfrak{A}$ onto $\mathfrak{B}, \mathfrak{A}$ and $\mathfrak{B}$ satisfy exactly the same equality-free sentences.

Given a class $K$ of $L$-structures, we denote by $\mathbf{H}_{S} K$ the class of all strict homomorphic images of members of $K$ and by $\mathbf{H}_{S}^{-1} K$ the class of all strict homomorphic pre-images of members of $K$.

Definition 2.3 Let $\mathfrak{A}$ be an $L$-structure and $B \subseteq A$, we define for any tuple $\bar{a}$ of elements of $A$ the equality-free type of $\bar{a}$ over $B$ in $\mathfrak{A}$ by

$$
\operatorname{tp}_{\mathfrak{A}}^{-}(\bar{a} / B)=\left\{\varphi(\bar{x}) \in L^{-}(B): \mathfrak{A}_{B} \models \varphi[\bar{a}]\right\},
$$

and the equality-free atomic type of $\bar{a}$ over $B$ in $\mathfrak{A}$ by

$$
\operatorname{atp}_{\mathfrak{A}}^{-}(\bar{a} / B)=\left\{\varphi(\bar{x}) \in \operatorname{tp}_{\mathfrak{A}}^{-}(\bar{a} / B): \varphi \text { is atomic }\right\} .
$$

Definition 2.4 Given a structure $\mathfrak{A}$, we define the relation $\Omega(\mathfrak{A})$ on $\mathfrak{A}$ by

$$
\Omega(\mathfrak{A})=\left\{\langle a, b\rangle \in A^{2}: \operatorname{atp}_{\mathfrak{A}}^{-}(a / A)=\operatorname{atp}_{\mathfrak{A}}^{-}(b / A)\right\} .
$$

This relation is a congruence of $\mathfrak{A}$ and it is called the Leibniz congruence of $\mathfrak{A}$. In fact the Leibniz congruence of $\mathfrak{A}$ is the greatest congruence relation on $\mathfrak{A}$ (i.e., it refines every congruence of $\mathfrak{A})$. Since for any $a, b \in A$,

$$
\operatorname{atp}_{\mathfrak{A}}^{-}(a / A)=\operatorname{atp}_{\mathfrak{A}}^{-}(b / A) \text { iff } \operatorname{tp}_{\mathfrak{A}}^{-}(a / A)=\operatorname{tp}_{\mathfrak{A}}^{-}(b / A)
$$

we have

$$
a \equiv b(\bmod \Omega(\mathfrak{A})) \quad \text { iff } \quad \operatorname{tp}_{\mathfrak{A}}^{-}(a / A)=\operatorname{tp}_{\mathfrak{A}}^{-}(b / A) .
$$

A structure is reduced if its Leibniz congruence is the identity. The quotient $\mathfrak{A} / \Omega(\mathfrak{A})$ is reduced and is called the reduction of $\mathfrak{A}$; it will be denoted by $\mathfrak{A}^{*}$. Notice that for any reduced structure $\mathfrak{A}, \mathfrak{A} \cong \mathfrak{A}^{*}$. Moreover, it is easy to check that the canonical homomorphism from $\mathfrak{A}$ onto $\mathfrak{A}^{*}$ is strict.

We now introduce an equivalence relation between structures that plays for languages without equality the same role that isomorphisms play for languages with equality. To our knowledge this relation was first defined by Blok and Pigozzi for the special case of logical matrices in [3], using Condition (ii) of Proposition 2.6 below as the defining condition; the word "relative" was introduced by them.

Definition 2.5 Let $\mathfrak{A}$ and $\mathfrak{B}$ be $L$-structures. We say that a relation $R \subseteq A \times B$ is a relativeness correspondence between $\mathfrak{A}$ and $\mathfrak{B}$ if $\operatorname{dom}(R)=A, \operatorname{rg}(R)=B$ and

(1) for any constant $c \in L, c^{\mathfrak{A}} R c^{\mathfrak{B}}$,

(2) for any $n$-ary function symbol $f \in L$, any $a_{1}, \ldots, a_{n} \in A$ and any $b_{1}, \ldots, b_{n} \in$ $B$ such that $a_{i} R b_{i}$ for each $i=1, \ldots, n$,

$$
f^{\mathfrak{A}}\left(a_{1}, \ldots, a_{n}\right) R f^{\mathfrak{B}}\left(b_{1}, \ldots, b_{n}\right),
$$


(3) for any $n$-ary relation symbol $P \in L$, any $a_{1}, \ldots, a_{n} \in A$ and any $b_{1}, \ldots, b_{n} \in$ $B$ such that $a_{i} R b_{i}$ for each $i=1, \ldots, n$,

$$
\left\langle a_{1}, \ldots, a_{n}\right\rangle \in P^{\mathfrak{A}} \text { iff }\left\langle b_{1}, \ldots, b_{n}\right\rangle \in P^{\mathfrak{B}} .
$$

And we say that two $L$-structures $\mathfrak{A}$ and $\mathfrak{B}$ are relatives, in symbols $\mathfrak{A} \sim \mathfrak{B}$, if there is a relativeness correspondence between them.

The relation of being either a strict homomorphic image or a strict homomorphic pre-image is not in general transitive. Its transitivization is precisely the relativeness relation, as the next proposition states. The equivalences between (ii), (iii), (iv) and (v) already appear in $[3$ for the special case of logical matrices.

Proposition 2.6 Let $\mathfrak{A}$ and $\mathfrak{B}$ be L-structures. Then the following are equivalent.

(i) $\mathfrak{A} \sim \mathfrak{B}$.

(ii) There are $n \in \omega$ and L-structures $\mathfrak{C}_{0}, \ldots, \mathfrak{C}_{n}$ such that $\mathfrak{A}=\mathfrak{C}_{0}, \mathfrak{B}=\mathfrak{C}_{n}$ and for any $i<n, \mathfrak{C}_{i+1} \in \mathbf{H}_{S}\left(\mathfrak{C}_{i}\right)$ or $\mathfrak{C}_{i+1} \in \mathbf{H}_{S}^{-1}\left(\mathfrak{C}_{i}\right)$.

(iii) $\mathfrak{A}, \mathfrak{B} \in \mathbf{H}_{S}(\mathfrak{C})$ for some $\mathfrak{C}$.

(iv) $\mathfrak{A}, \mathfrak{B} \in \mathbf{H}_{S}^{-1}(\mathfrak{C})$ for some $\mathfrak{C}$.

(v) $\mathfrak{A}^{*} \cong \mathfrak{B}^{*}$.

(vi) There are enumerations of $A$ and $B, \bar{a}=\left(a_{i}: i \in I\right)$ and $\bar{b}=\left(b_{i}: i \in I\right)$ respectively, such that $(\mathfrak{A}, \bar{a}) \equiv_{0}^{-}(\mathfrak{B}, \bar{b})$.

(vii) There are enumerations of $A$ and $B, \bar{a}=\left(a_{i}: i \in I\right)$ and $\bar{b}=\left(b_{i}: i \in I\right) \mathrm{re}$ spectively, such that $(\mathfrak{A}, \bar{a}) \equiv^{-}(\mathfrak{B}, \bar{b})$.

Proof: $\quad$ It is clear that (vi) $\Leftrightarrow$ (vii). The directions (iii) $\Rightarrow$ (ii), (iv) $\Rightarrow$ (ii) and (v) $\Rightarrow$ (iv) are also clear.

(vi) $\Rightarrow$ (v) Suppose that there are enumerations of $A$ and $B, \bar{a}=\left(a_{i}: i \in I\right)$ and $\bar{b}=\left(b_{i}: i \in I\right)$, respectively, such that

$$
(\mathfrak{A}, \bar{a}) \equiv_{0}^{-}(\mathfrak{B}, \bar{b}) .
$$

We define $h: \mathfrak{A}^{*} \rightarrow \mathfrak{B}^{*}$ as follows: for any $i \in I$

$$
h\left(\left[a_{i}\right]_{\Omega(\mathfrak{A})}\right)=\left[b_{i}\right]_{\Omega(\mathfrak{B})} .
$$

First of all we show that for any term $t\left(y_{1}, \ldots, y_{n}\right)$ of $L$ and any $i_{1}, \ldots, i_{n}, j \in I$,

$$
\left[t^{\mathfrak{A}}\left[a_{i_{1}}, \ldots, a_{i_{n}}\right]\right]_{\Omega(\mathfrak{A})}=\left[a_{j}\right]_{\Omega(\mathfrak{A})} \text { iff }\left[t^{\mathfrak{B}}\left[b_{i_{1}}, \ldots, b_{i_{n}}\right]\right]_{\Omega(\mathfrak{B})}=\left[b_{j}\right]_{\Omega(\mathfrak{B})} .
$$

Assume that

$$
\left[t^{\mathfrak{A}}\left[a_{i_{1}}, \ldots, a_{i_{n}}\right]\right]_{\Omega(\mathfrak{A})}=\left[a_{j}\right]_{\Omega(\mathfrak{A})}
$$

but

$$
\left[t^{\mathfrak{B}}\left[b_{i_{1}}, \ldots, b_{i_{n}}\right]\right]_{\Omega(\mathfrak{B})} \neq\left[b_{j}\right]_{\Omega(\mathfrak{B})} .
$$

Then, there is some quantifier-free formula $\varphi\left(z, x_{1}, \ldots, x_{m}\right) \in L^{-}$(where the variables $z, x_{1}, \ldots, x_{m}$ don't occur in $t$ ) and a sequence $d_{1}, \ldots, d_{m}$ of elements of $B$ such that

$$
\mathfrak{B} \models \varphi\left(z, x_{1}, \ldots, x_{m}\right)\left[t^{\mathfrak{B}}\left[b_{i_{1}}, \ldots, b_{i_{n}}\right], d_{1}, \ldots, d_{m}\right],
$$


but

$$
\mathfrak{B} \not \models \varphi\left(z, x_{1}, \ldots, x_{m}\right)\left[b_{j}, d_{1}, \ldots, d_{m}\right] .
$$

Then, for any $0<k \leq m$, we choose $j_{k} \in I$ such that $d_{k}=b_{j_{k}}$ in the enumeration $\bar{b}$ of $B$. Hence,

$$
\mathfrak{B} \models \varphi\left(z, x_{1}, \ldots, x_{m}\right)\left[t^{\mathfrak{B}}\left[b_{i_{1}}, \ldots, b_{i_{n}}\right], b_{j_{1}}, \ldots, b_{j_{m}}\right],
$$

but

$$
\mathfrak{B} \not \models \varphi\left(z, x_{1}, \ldots, x_{m}\right)\left[b_{j}, b_{j_{1}}, \ldots, b_{j_{m}}\right] .
$$

Let $\varphi^{\prime}$ be obtained from $\varphi$ by substituting the term $t$ for the variable $z$. We have

$$
\mathfrak{B} \models \varphi^{\prime}\left(y_{1}, \ldots, y_{n}, x_{1}, \ldots, x_{m}\right)\left[b_{i_{1}}, \ldots, b_{i_{n}}, b_{j_{1}}, \ldots, b_{j_{m}}\right] .
$$

Since

$$
(\mathfrak{A}, \bar{a}) \equiv_{0}^{-}(\mathfrak{B}, \bar{b})
$$

we have

$$
\mathfrak{A} \models \varphi^{\prime}\left(y_{1}, \ldots, y_{n}, x_{1}, \ldots, x_{m}\right)\left[a_{i_{1}}, \ldots, a_{i_{n}}, a_{j_{1}}, \ldots, a_{j_{m}}\right]
$$

and

$$
\mathfrak{A} \not \models \varphi\left(z, x_{1}, \ldots, x_{m}\right)\left[a_{j}, a_{j_{1}}, \ldots, a_{j_{m}}\right] .
$$

But then

$$
\mathfrak{A} \models \varphi\left(z, x_{1}, \ldots, x_{m}\right)\left[t^{\mathfrak{A}}\left[a_{i_{1}}, \ldots, a_{i_{n}}\right], a_{j_{1}}, \ldots, a_{j_{m}}\right],
$$

which is absurd. Therefore, we conclude that

$$
\left[t^{\mathfrak{B}}\left[b_{i_{1}}, \ldots, b_{i_{n}}\right]\right]_{\Omega(\mathfrak{B})}=\left[b_{j}\right]_{\Omega(\mathfrak{B})} .
$$

We can prove the other direction of (1) analogously. By using 11 it is easy to see that $h$ is well-defined and it is an isomorphism.

(ii) $\Rightarrow$ (v) It suffices to show that if $\mathfrak{A}$ and $\mathfrak{B}$ are $L$-structures and $h: \mathfrak{A} \rightarrow \mathfrak{B}$ is a strict homomorphism from $\mathfrak{A}$ onto $\mathfrak{B}$ then $\mathfrak{A}^{*} \cong \mathfrak{B}^{*}$. Since $h$ is onto $\mathfrak{B}$, we have that $\bar{a}=(a: a \in A)$ and $\bar{b}=(h(a): a \in A)$ are enumerations (possibly with repetitions) of $A$ and $B$. And since $h$ is a strict homomorphism, by Lemma 2.2. we obtain that

$$
(\mathfrak{A}, \bar{a}) \equiv_{0}^{-}(\mathfrak{B}, \bar{b}) .
$$

Therefore, by the implication (vi) $\Rightarrow(\mathrm{v})$, we conclude that $\mathfrak{A}^{*} \cong \mathfrak{B}^{*}$.

(vi) $\Rightarrow$ (iii) Suppose that there are enumerations $\bar{a}=\left(a_{i}: i \in I\right)$ and $\bar{b}=\left(b_{i}\right.$ : $i \in I$ ) of $A$ and $B$ respectively such that

$$
(\mathfrak{A}, \bar{a}) \equiv_{0}^{-}(\mathfrak{B}, \bar{b}) .
$$

Let $V=\left\{x_{i}: i \in I\right\}$ be a set of variables, $\mathcal{T}$ the algebra of terms of type $L$ freely generated by the set $V$ and $h$ the homomorphism from $\mathcal{T}$ onto the algebraic reduct of $\mathfrak{A}$ such that for any $i \in I$

$$
h\left(x_{i}\right)=a_{i}
$$


We define an $L$-structure $\mathfrak{C}$ in the following way. The algebraic reduct of $\mathfrak{C}$ is $\mathcal{T}$ and for any $n$-ary relation symbol $R \in L$ and any $t_{1}, \ldots, t_{n} \in \mathcal{T}$

$$
\left\langle t_{1}, \ldots, t_{n}\right\rangle \in R^{\mathfrak{C}} \text { iff }\left\langle h\left(t_{1}\right), \ldots, h\left(t_{n}\right)\right\rangle \in R^{\mathfrak{A}} .
$$

It is easy to see that $h$ is a strict homomorphism from $\mathfrak{C}$ onto $\mathfrak{A}$. And since

$$
(\mathfrak{A}, \bar{a}) \equiv_{0}^{-}(\mathfrak{B}, \bar{b}),
$$

the function $f_{0}: V \rightarrow B$ such that for any $i \in I$

$$
f_{0}\left(x_{i}\right)=b_{i}
$$

can be extended to a strict homomorphism $f$ from $\mathfrak{C}$ onto $\mathfrak{B}$. Therefore $\mathfrak{A} \in \mathbf{H}_{S}(\mathfrak{C})$ and $\mathfrak{B} \in \mathbf{H}_{S}(\mathfrak{C})$.

(i) $\Rightarrow$ (vi) Let $R$ be a relativeness correspondence between $\mathfrak{A}$ and $\mathfrak{B}$. An easy induction shows that for any equality and quantifier-free formula $\varphi\left(x_{1}, \ldots, x_{n}\right)$ of $L$, any $a_{1}, \ldots, a_{n} \in A$ and any $b_{1}, \ldots, b_{n} \in B$ such that for each $i, 1 \leq i \leq n, a_{i} R b_{i}$,

$$
\mathfrak{A} \models \varphi\left[a_{1}, \ldots, a_{n}\right] \text { iff } \mathfrak{B} \models \varphi\left[b_{1}, \ldots, b_{n}\right] .
$$

Therefore, if there are enumerations $\bar{a}=\left(a_{i}: i \in I\right)$ and $\bar{b}=\left(b_{i}: i \in I\right)$ of $A$ and $B$ respectively such that for any $i \in I, a_{i} R b_{i}$, we have $(\mathfrak{A}, \bar{a}) \equiv_{0}^{-}(\mathfrak{B}, \bar{b})$. Clearly such enumerations exist.

(v) $\Rightarrow$ (i) Assume that $\mathfrak{A}^{*} \cong \mathfrak{B}^{*}$ and let $h: \mathfrak{A}^{*} \rightarrow \mathfrak{B}^{*}$ be an isomorphism. Define the relation $R \subseteq A \times B$ by

$$
a R b \text { iff } h\left([a]_{\Omega(\mathfrak{A})}\right)=[b]_{\Omega(\mathfrak{B})},
$$

for any $a \in A$ and any $b \in B$. It is easy to check that $R$ is a relativeness correspondence between $\mathfrak{A}$ and $\mathfrak{B}$.

Lemma 2.7 Let $\mathfrak{A}$ and $\mathfrak{B}$ be L-structures and suppose that there are sequences of elements of $A$ and $B, \bar{a}=\left(a_{i}: i \in I\right)$ and $\bar{b}=\left(b_{i}: i \in I\right)$, respectively, such that

$$
(\mathfrak{A}, \bar{a}) \equiv^{-}(\mathfrak{B}, \bar{b}) .
$$

Then there are $\mathfrak{A}^{\prime} \succ \mathfrak{A}$ and sequences $\bar{c}=\left(a_{j}: j \in J\right)$ and $\bar{d}=\left(b_{j}: j \in J\right)$ of elements of $A^{\prime}$ and $B$, respectively, such that

$$
\left(\mathfrak{A}^{\prime}, \bar{c}\right) \equiv^{-}(\mathfrak{B}, \bar{d}),
$$

$\bar{a} \subseteq \bar{c}, \bar{b} \subseteq \bar{d}$ and $\bar{d}$ is an enumeration of $B$.

Proof: We expand the language introducing new constants classified in the following three disjoint sets

$$
C_{A}=\left\{c_{a}: a \in A-\operatorname{rg}(\bar{a})\right\}, C_{I}=\left\{c_{i}: i \in I\right\}, C_{B}=\left\{c_{b}: b \in B-\operatorname{rg}(\bar{b})\right\},
$$

and we consider the elementary diagram of $\mathfrak{A}$ in this expanded language, i.e., the set of all sentences of type $L \cup C_{I} \cup C_{A}$ true in $(\mathfrak{A}, \bar{a}, a)_{a \in A-\operatorname{rg}(\bar{a})}$, and the equality-free 
elementary diagram of $\mathfrak{B}$ in this expanded language, i.e., the set of all equality-free sentences of type $L \cup C_{I} \cup C_{B}$ true in $(\mathfrak{B}, \bar{b}, b)_{b \in B-\operatorname{rg}(\bar{b})}$. Let $\Gamma$ be the union of these two diagrams. Since $(\mathfrak{A}, \bar{a}) \equiv^{-}(\mathfrak{B}, \bar{b}), \Gamma$ is consistent. Let

$$
\mathfrak{C}=\left(\mathfrak{A}^{\prime}, c^{\mathfrak{C}}\right)_{c \in C_{A} \cup C_{B} \cup C_{I}}
$$

be a model of $\Gamma$. We may assume that $c_{i}^{\mathfrak{C}}=a_{i}$ for all $i \in I$ and that $c_{a}^{\mathfrak{C}}=a$ for all $a \in A-\operatorname{rg}(\bar{a})$. Since $\mathfrak{C}$ is a model of the elementary diagram of $\mathfrak{A}$ we have that $\mathfrak{A} \prec$ $\mathfrak{A}^{\prime}$. Let $\bar{c}=\bar{a} \cup\left(c_{b}^{\mathfrak{C}}: b \in B-\operatorname{rg}(\bar{b})\right)$ and $\bar{d}=\bar{b} \cup(b: b \in B-\operatorname{rg}(\bar{b}))$. Since $\mathfrak{C}^{\mathfrak{C}}$ is a model of the equality-free elementary diagram of $\mathfrak{B}$,

$$
\left(\mathfrak{A}^{\prime}, \bar{c}\right) \equiv^{-}(\mathfrak{B}, \bar{d}) .
$$

Theorem 2.8 Let $\mathfrak{A}$ and $\mathfrak{B}$ be L-structures. Then the following are equivalent.

(i) $\mathfrak{A} \equiv{ }^{-} \mathfrak{B}$.

(ii) There are $\mathfrak{C} \succ \mathfrak{A}$ and $\mathfrak{D} \succ \mathfrak{B}$ such that $\mathfrak{C} \sim \mathfrak{D}$.

Proof: (ii) $\Rightarrow$ (i) is clear. (i) $\Rightarrow$ (ii) Suppose that $\mathfrak{A} \equiv^{-} \mathfrak{B}$. Using Lemma 2.7 we can define by induction two elementary chains of models $\left(\mathfrak{A}_{n}\right)_{n \in \omega}$ and $\left(\mathfrak{B}_{n}\right)_{n \in \omega}$ and two chains of sequences $\left(\bar{a}_{n}\right)_{n \in \omega}$ and $\left(\bar{b}_{n}\right)_{n \in \omega}$ such that $\mathfrak{A}_{0}=\mathfrak{A}, \mathfrak{B}_{0}=\mathfrak{B}$ and for any $n \in \omega:$

a) $\bar{a}_{n}=\left(a_{i}: i \in I_{n}\right)$ and $\bar{b}_{n}=\left(b_{i}: i \in I_{n}\right)$ are sequences of elements of $A_{n}$ and $B_{n}$ respectively such that

$$
\left(\mathfrak{A}_{n}, \bar{a}_{n}\right) \equiv^{-}\left(\mathfrak{B}_{n}, \bar{b}_{n}\right)
$$

and

b) $A_{n} \subseteq \operatorname{rg}\left(\bar{a}_{n+1}\right)$ and $B_{n} \subseteq \operatorname{rg}\left(\bar{b}_{n+1}\right)$.

Let $\mathfrak{C}=\bigcup_{n \in \omega} \mathfrak{A}_{n}, \mathfrak{D}=\bigcup_{n \in \omega} \mathfrak{B}_{n}, \bar{c}=\bigcup_{n \in \omega} \bar{a}_{n}$ and $\bar{d}=\bigcup_{n \in \omega} \bar{b}_{n}$. We have that $\mathfrak{C} \succ \mathfrak{A}$ and $\mathfrak{D} \succ \mathfrak{B}$. Moreover, $\bar{c}$ and $\bar{d}$ are enumerations of $C$ and $D$, respectively, and

$$
(\mathfrak{C}, \bar{c}) \equiv^{-}(\mathfrak{D}, \bar{d}) .
$$

By Proposition 2.6. we conclude that $\mathfrak{C} \sim \mathfrak{D}$.

Theorem 2.9 Let $K$ be a class of $L$-structures. The following are equivalent.

(i) $K$ is axiomatizable by a set of equality-free sentences.

(ii) $K$ is closed under ultraproducts, $\mathbf{H}_{S}$ and $\mathbf{H}_{S}^{-1}$ and for any L-structure $\mathfrak{A}$ the following holds: if some ultrapower of $\mathfrak{A}$ lies in $K$, then $\mathfrak{A} \in K$.

Proof: (i) $\Rightarrow$ (ii) is clear. (ii) $\Rightarrow$ (i) Consider the equality-free theory of $K$ :

$\mathrm{Th}^{-}(K)=\left\{\sigma: \sigma\right.$ is a sentence of $L^{-}$and for any $\left.\mathfrak{B} \in K, \mathfrak{B} \models \sigma\right\}$.

We will show that if $\mathfrak{A} \models \mathrm{Th}^{-}(K)$ then $\mathfrak{A} \in K$. Suppose that $\mathfrak{A} \models \mathrm{Th}^{-}(K)$. Let $\mathrm{Th}^{-}(\mathfrak{A})$ be the equality-free theory of $\mathfrak{A}$ :

$$
\mathrm{Th}^{-}(\mathfrak{A})=\left\{\sigma: \sigma \text { is a sentence of } L^{-} \text {and } \mathfrak{A} \models \sigma\right\} .
$$


Since $\mathfrak{A} \models \mathrm{Th}^{-}(K)$, for any $\sigma \in \mathrm{Th}^{-}(\mathfrak{A})$ there is $\mathfrak{B}_{\sigma} \in K$ such that $\mathfrak{B}_{\sigma} \models \sigma$. Let $I=\mathrm{Th}^{-}(\mathfrak{A})$ and consider for any $\sigma \in \mathrm{Th}^{-}(\mathfrak{A})$ the set $J_{\sigma}=\{\beta \in I: \beta \models \sigma\}$. Since $J=\left\{J_{\sigma}: \sigma \in I\right\}$ has the finite intersection property it can be extended to an ultrafilter $U$ over $I$. Let $\mathfrak{B}=\prod_{\sigma \in I} \mathfrak{B}_{\sigma} / U$. Observe that $\mathfrak{A} \equiv^{-} \mathfrak{B}$. Since $K$ is closed under ultraproducts, $\mathfrak{B} \in K$. By Theorem 2.8 there are $\mathfrak{C} \succ \mathfrak{A}$ and $\mathfrak{D} \succ \mathfrak{B}$ such that $\mathfrak{C} \sim \mathfrak{D}$. Therefore, by Proposition 2.6. $\mathfrak{C}^{*} \cong \mathfrak{D}^{*}$. By the assumption we know that $K$ is an elementary class (see [7] p. 322) and therefore, since $\mathfrak{B} \in K$ and $\mathfrak{D} \succ \mathfrak{B}$, we have $\mathfrak{D} \in K$. Since $K$ is closed under $\mathbf{H}_{S}, \mathfrak{D}^{*} \in K$. Consequently, $\mathfrak{C}^{*} \in K$ and since $K$ is closed under $\mathbf{H}_{S}^{-1}, \mathfrak{C} \in K$. Since $\mathfrak{C} \succ \mathfrak{A}$ we conclude that $\mathfrak{A} \in K$.

Corollary 2.10 Let $T \cup\{\sigma\}$ be a set of sentences of type $L$. Then

(i) $T$ is axiomatizable by a set of equality-free sentences iff $T$ is preserved under $\mathbf{H}_{S}$ and $\mathbf{H}_{S}^{-1}$

(ii) $\sigma$ is logically equivalent to an equality-free sentence iff $\sigma$ is preserved under $\mathbf{H}_{S}$ and $\mathbf{H}_{S}^{-1}$.

Proof: (i) The implication from left to right is clear. In order to prove the other implication note that since $T$ is a set of sentences, $\operatorname{Mod}(T)$ is closed under ultraproducts and if some ultrapower of $\mathfrak{A}$ lies in $\operatorname{Mod}(T), \mathfrak{A} \in \operatorname{Mod}(T)$. Moreover, since $T$ is preserved under $\mathbf{H}_{S}$ and $\mathbf{H}_{S}^{-1}, \operatorname{Mod}(T)$ is closed under $\mathbf{H}_{S}$ and $\mathbf{H}_{S}^{-1}$. By Theorem 2.9. $T$ can be axiomatized by a set of equality-free sentences.

(ii) The implication from left to right is clear. We prove the other implication. By (i) there is a set of equality-free sentences $\Gamma$ such that $\operatorname{Mod}(\Gamma)=\operatorname{Mod}(\sigma)$. By compactness, there is a finite $\Gamma_{0} \subseteq \Gamma$ such that $\Gamma_{0} \models \sigma$. Then $\sigma$ is logically equivalent to $\bigwedge \Gamma_{0}$.

3 Ultrapower-type characterizations of $\equiv^{-} \quad$ There is a well-known characterization of elementary equivalence in terms of ultrapowers due to Keisler and Shelah according to which two structures $\mathfrak{A}$ and $\mathfrak{B}$ are elementarily equivalent iff there is an ultrafilter $U$ over a set of power $\leq 2^{|A|+|B|+\omega}$ such that $\mathfrak{A}^{U} \cong \mathfrak{B}^{U}$. A similar characterization holds for elementary equivalence for equality-free logic if instead of isomorphism of ultrapowers only relativeness of ultrapowers is postulated.

Theorem 3.1 If $\mathfrak{A}$ and $\mathfrak{B}$ are L-structures, the following are equivalent.

(i) $\mathfrak{A} \equiv{ }^{-} \mathfrak{B}$.

(ii) $\mathfrak{A}^{U} \sim \mathfrak{B}^{U}$ for some ultrafilter $U$ over a set of power $\leq 2^{|A|+|B|+\omega}$.

Proof: $\quad$ Let $\kappa=2^{|A|+|B|+\omega}$. Then $|A|^{\kappa},|B|^{\kappa} \leq 2^{\kappa}$. We may assume that $|L| \leq \kappa$. In the proof of the analogous result for first-order languages with equality in Shelah [10] (the interested reader may also consult $[7$ ), it is shown under the hypothesis that $\mathfrak{A} \equiv$ $\mathfrak{B}$ that there are enumerations $\left(a_{i}: i<2^{\kappa}\right)$ and $\left(b_{i}: i<2^{\kappa}\right)$ of ${ }^{\kappa} A$ and ${ }^{\kappa} B$ respectively and an ultrafilter $U$ over $\kappa$ such that for any first-order formula $\varphi\left(x_{1}, \ldots, x_{n}\right)$ and any $i_{1}<\cdots<i_{n}<2^{\kappa}$,

$\left\{j<\kappa: \mathfrak{A}=\varphi\left[a_{i_{1}}(j), \ldots, a_{i_{n}}(j)\right]\right\} \in U$ iff $\left\{j<\kappa: \mathfrak{B} \models \varphi\left[b_{i_{1}}(j), \ldots, b_{i_{n}}(j)\right]\right\} \in U$. 
The very same proof works for an equality-free $\varphi$ under the sole hypothesis that $\mathfrak{A} \equiv^{-}$ $\mathfrak{B}$. In the case of a language with equality the conclusion is

$$
\left(\mathfrak{A}^{U},\left(\left[a_{i}\right]_{U}\right)_{i<2^{\kappa}}\right) \equiv\left(\mathfrak{B}^{U},\left(\left[b_{i}\right]_{U}\right)_{i<2^{\kappa}}\right)
$$

and therefore, $\mathfrak{A}^{U} \cong \mathfrak{B}^{U}$. In the case of an equality-free language we may conclude that

$$
\left(\mathfrak{A}^{U},\left(\left[a_{i}\right]_{U}\right)_{i<2^{\kappa}}\right) \equiv^{-}\left(\mathfrak{B}^{U},\left(\left[b_{i}\right]_{U}\right)_{i<2^{\kappa}}\right)
$$

and by Proposition 2.6 that $\mathfrak{A}^{U} \sim \mathfrak{B}^{U}$.

Notice that, by Proposition 2.6. Theorem 3.1 can be rephrased as follows.

$$
\mathfrak{A} \equiv^{-} \mathfrak{B} \text { iff }\left(\mathfrak{A}^{U}\right)^{*} \cong\left(\mathfrak{B}^{U}\right)^{*},
$$

for some ultrafilter $U$ over a set of power $\leq 2^{|A|+|B|+\omega}$.

The composition of the ultrapower operation with the reduction operation is a quotient of the direct product and we may consider it as a single operation. This quotient operation is what actually plays the role in equality-free logic that the ultrapower operation plays in the Keisler-Shelah theorem.

In equality-free logic the reduced product, the ultraproduct and the ultrapower operators are not the most natural ones because there is no need to consider quotients modulo the relation associated to the filter. We now introduce some operators that play in equality-free logic the same role that ultraproducts and ultrapowers play in logic with equality. They have been considered, for example, by Monk 9 and Blok and Pigozzi [5, but to our view their role in equality-free logic has not been stressed enough.

Definition 3.2 Let $I$ be a nonempty set, $\left(\mathfrak{A}_{i}\right)_{i \in I}$ a family of $L$-structures and $U$ an ultrafilter over $I$. We define the ultrafilter-product of the family $\left(\mathfrak{A}_{i}\right)_{i \in I}$ modulo $U$, that we denote by $\prod_{i \in I}^{U} \mathfrak{A}_{i}$, as follows.

- The domain of $\prod_{i \in I}^{U} \mathfrak{A}_{i}$ is $\prod_{i \in I} A_{i}$.

- For any constant $c \in L, c \prod_{i \in I}^{U} \mathfrak{A}_{i}=\left\langle c^{\mathfrak{A}_{i}}: i \in I\right\rangle$.

- For any $n$-ary function symbol $f \in L$ and any $a_{1}, \ldots, a_{n} \in \prod_{i \in I} A_{i}$,

$$
f^{\prod_{i \in I}^{U} \mathfrak{A}_{i}}\left(a_{1}, \ldots, a_{n}\right)=\left(f^{\mathfrak{A}_{i}}\left(a_{1}(i), \ldots, a_{n}(i)\right): i \in I\right) .
$$

- For any $n$-ary relation symbol $R \in L$ and any $a_{1}, \ldots, a_{n} \in \prod_{i \in I} A_{i}$,

$$
\left\langle a_{1}, \ldots, a_{n}\right\rangle \in R^{\prod_{i \in I}^{U} \mathfrak{A}_{i}} \text { iff }\left\{i \in I:\left\langle a_{1}(i), \ldots, a_{n}(i)\right\rangle \in R^{\mathfrak{A}_{i}}\right\} \in U .
$$

When for any $i \in I, \mathfrak{A}_{i}=\mathfrak{A}$, we say that $\prod^{U} \mathfrak{A}$ is the ultrafilter-power of $\mathfrak{A}$. Note that the relation $\sim_{U}$ defined on $\prod_{i \in I} A_{i}$ by

$$
a \sim_{U} b \text { iff }\{i \in I: a(i)=b(i)\} \in U
$$

is a congruence relation of $\prod_{i \in I}^{U} \mathfrak{A}_{i}$ and the ultraproduct $\prod_{i \in I} \mathfrak{A}_{i} / U$ is precisely the quotient $\prod_{i \in I}^{U} \mathfrak{A}_{i} / \sim_{U}$. Therefore $\prod_{i \in I}^{U} \mathfrak{A}_{i} \in \mathbf{H}_{S}^{-1}\left(\prod_{i \in I} \mathfrak{A}_{i} / U\right)$ and so $\prod_{i \in I}^{U} \mathfrak{A}_{i}$ and $\prod_{i \in I} \mathfrak{A}_{i} / U$ are relatives. This shows that ultraproducts are not necessary in equalityfree logic, and allows us to rephrase Theorem 3.1 and Theorem 2.9 in the following way. 
Theorem 3.3 If $\mathfrak{A}$ and $\mathfrak{B}$ are L-structures, the following are equivalent:

(i) $\mathfrak{A} \equiv{ }^{-} \mathfrak{B}$;

(ii) $\prod^{U} \mathfrak{A} \sim \prod^{U} \mathfrak{B}$ for some ultrafilter $U$ over a set of power $\leq 2^{|A|+|B|+\omega}$.

Proof: By Theorem 3.1 .

Theorem 3.4 Let $K$ be a class of L-structures. Then the following are equivalent.

(i) $K$ is axiomatizable by a set of equality-free sentences;

(ii) $K$ is closed under ultrafilter-products, $\mathbf{H}_{S}$ and $\mathbf{H}_{S}^{-1}$ and for any L-structure $\mathfrak{A}$ the following holds: if some ultrafilter-power of $\mathfrak{A}$ lies in $K$, then $\mathfrak{A} \in K$.

Proof: By Theorem 2.9.

We now state some easy facts about the new constructions. The next proposition is the version of Łoś theorem for equality-free logic and the ultrafilter-product construction, and its proof is straightforward.

Proposition 3.5 Let I be a nonempty set, $\left(\mathfrak{A}_{i}\right)_{i \in I}$ a family of L-structures and $U$ an ultrafilter over $I$. Then, for any $a_{1}, \ldots, a_{n} \in \prod_{i \in I} A_{i}$ and any formula $\varphi\left(x_{1}, \ldots, x_{n}\right)$ $\in L^{-}$,

$$
\prod_{i \in I}^{U} \mathfrak{A}_{i} \models \varphi\left[a_{1}, \ldots, a_{n}\right] \text { iff }\left\{i \in I: \mathfrak{A}_{i} \models \varphi\left[a_{1}(i), \ldots, a_{n}(i)\right]\right\} \in U .
$$

The notion of elementary substructure can be generalized to equality-free logic in a natural way. If $\mathfrak{A}$ and $\mathfrak{B}$ are $L$-structures, we say that $\mathfrak{A}$ is an $L^{-}$-substructure of $\mathfrak{B}$, written $\mathfrak{A} \prec^{-} \mathfrak{B}$, if $\mathfrak{A} \subseteq \mathfrak{B}$ and for any equality-free formula $\varphi\left(x_{1}, \ldots, x_{n}\right)$ of $L$ and any $a_{1}, \ldots, a_{n} \in A$,

$$
\mathfrak{A} \models \varphi\left[a_{1}, \ldots, a_{n}\right] \text { iff } \mathfrak{B} \models \varphi\left[a_{1}, \ldots, a_{n}\right] .
$$

It clearly holds that if $U$ is an ultrafilter over $I$, the mapping $h(a)=(a: i \in I)$ is an isomorphism of $\mathfrak{A}$ onto an $L^{-}$-substructure of $\prod^{U} \mathfrak{A}$.

The next example shows that in Theorem 3.3 we can not replace the relativeness relation by isomorphism, that is, it shows that it is not true in general that $\mathfrak{A} \equiv^{-} \mathfrak{B}$ implies that there is an ultrafilter $U$ such that $\prod^{U} \mathfrak{A} \cong \prod^{U} \mathfrak{B}$. Note that this is not immediate since from $\prod^{U} \mathfrak{A} \cong \prod^{U} \mathfrak{B}$ we can not infer that $\mathfrak{A}^{U} \cong \mathfrak{B}^{U}$.

Example 3.6 Let $L$ be any similarity type with one monadic relation symbol $P$, one monadic function symbol $f$ (and possibly more function symbols but no more relation symbols). Let $\mathfrak{A}=\left(\{0,1\}, P^{\mathfrak{A}}, f^{\mathfrak{A}}, \ldots\right)$ and $\mathfrak{B}=\left(\{0,1\}, P^{\mathfrak{B}}, f^{\mathfrak{B}}, \ldots\right)$ be two $L$-structures with $P^{\mathfrak{A}}=P^{\mathfrak{B}}=\{0,1\}, f^{\mathfrak{A}}=\{\langle 0,0\rangle,\langle 1,1\rangle\}$ and $f^{\mathfrak{B}}=\{\langle 0,1\rangle,\langle 1,0\rangle\}$. Clearly $\mathfrak{A}^{*} \cong \mathfrak{B}^{*}$ and therefore $\mathfrak{A} \equiv \equiv^{-} \mathfrak{B}$. But there is no ultrafilter $U$ such that $\prod^{U} \mathfrak{A} \cong \prod^{U} \mathfrak{B}$, because $\prod^{U} \mathfrak{A} \models \forall x f(x)=x$ and $\prod^{U} \mathfrak{B} \not \forall \forall x f(x)=x$.

Now we see that for relational similarity types and structures with at least two elements we can indeed replace in Theorem 3.3 the relation of relativeness by the isomorphism one.

Lemma 3.7 Let $\mathfrak{A}$ be an L-structure with at least two elements, I a nonempty set and $U$ an ultrafilter over I. Then, for any $a \in A^{I},|U| \leq\left|[a]_{\Omega\left(\Pi^{U} \mathfrak{A}\right)}\right|$. 
Proof: $\quad$ Let $a \in A^{I}$. We fix two distinct elements $c, d \in A$ and define for any $X \in U$ an element $a_{X} \in A^{I}$ in the following way.

$$
a_{X}(i)= \begin{cases}a(i), & \text { if } i \in X \\ c, & \text { if } i \notin X \text { and } a(i) \neq c \\ d, & \text { if } i \notin X \text { and } a(i)=c,\end{cases}
$$

for any $i \in I$. Clearly $\left\{i \in I: a_{X}(i)=a(i)\right\}=X \in U$. Therefore, since the relation $\sim_{U}$ in $A^{I}$, defined as before, is a congruence relation of $\prod^{U} \mathfrak{A}$ and the Leibniz congruence $\Omega\left(\prod^{U} \mathfrak{A}\right)$ is the greatest one, we have that $a_{X} \equiv a\left(\bmod \Omega\left(\prod^{U} \mathfrak{A}\right)\right)$. Moreover, for any $X, Y \in U$, if $X \neq Y$ we have that $a_{X} \neq a_{Y}$. Therefore we conclude that $|U| \leq\left|[a]_{\Omega\left(\prod^{U} \mathfrak{A}\right)}\right|$.

Theorem 3.8 Let $L$ be a relational similarity type and $\mathfrak{A}$ and $\mathfrak{B}$ two L-structures with at least two elements. Then the following are equivalent.

(i) $\mathfrak{A} \equiv{ }^{-} \mathfrak{B}$.

(ii) $\prod^{U} \mathfrak{A} \cong \prod^{U} \mathfrak{B}$ for some ultrafilter $U$ over a set of power $\leq 2^{|A|+|B|+\omega}$.

Proof: (ii) $\Rightarrow$ (i) is clear. (i) $\Rightarrow$ (ii) Suppose that $\mathfrak{A} \equiv^{-} \mathfrak{B}$. By Theorem 3.3 . $\prod^{U} \mathfrak{A} \sim \prod^{U} \mathfrak{B}$ for some ultrafilter $U$ over a set $I$ of power $\leq 2^{|A|+|B|+\omega}$. Without loss of generality we can assume that $|I| \geq|A|+|B|$ which implies that for every element $a \in A^{I}$ the cardinality of its equivalence class modulo the Leibniz congruence is $\leq|U|$, and similarly for every $b \in \mathfrak{B}^{I}$. By Lemma 3.7. we have that for any $a \in A^{I}$ and for any $b \in B^{I}$,

$$
(*) \quad\left|[a]_{\Omega\left(\Pi^{U} \mathfrak{A}\right)}\right|=|U|=\left|[b]_{\Omega\left(\Pi^{U} \mathfrak{B}\right)}\right| .
$$

Now since $\left(\prod^{U} \mathfrak{A}\right)^{*} \cong\left(\prod^{U} \mathfrak{B}\right)^{*}$, let $h$ be an isomorphism between these structures. With its help and using condition $(*)$ we obtain enumerations without repetitions of $A^{I}$ and $B^{I}, \bar{a}=\left(a_{j}: j \in J\right)$ and $\bar{b}=\left(b_{j}: j \in J\right)$, respectively, such that

$$
\left(\prod^{U} \mathfrak{A}, \bar{a}\right) \equiv^{-}\left(\prod^{U} \mathfrak{B}, \bar{b}\right) .
$$

Since $L$ is relational, the mapping sending $a_{j}$ to $b_{j}$ is an isomorphism from $\prod^{U} \mathfrak{A}$ onto $\prod^{U} \mathfrak{B}$.

Now we prove a more general version of the previous theorem. Notice that if $\mathfrak{A}$ is a one-element structure and $\mathfrak{A} \equiv^{-} \mathfrak{B}$, then $\mathfrak{A} \cong \mathfrak{B}^{*}$.

Corollary 3.9 Let $L$ be relational and let $\mathfrak{A}$ and $\mathfrak{B}$ be L-structures. Then, $\mathfrak{A} \equiv^{-} \mathfrak{B}$ if and only if one of the following three cases holds.

(i) $\prod^{U} \mathfrak{A} \cong \prod^{U} \mathfrak{B}$ for some ultrafilter $U$ over a set of power $\leq 2^{|A|+|B|+\omega}$.

(ii) $\mathfrak{A} \cong \mathfrak{B}^{*}$.

(iii) $\mathfrak{A}^{*} \cong \mathfrak{B}$.

Proof: Each one of the conditions (i), (ii) and (iii) implies that $\mathfrak{A} \equiv^{-} \mathfrak{B}$. Now, if $\mathfrak{A} \equiv \equiv^{-} \mathfrak{B}$ and both structures have at least two elements, condition (i) follows from the preceding theorem. In the case that one of the structures is a one-element structure, by the previous observation one of the other conditions follows. 
Corollary 3.9 allows us to obtain a new characterization of elementary classes in equality-free logic, although restricted to relational similarity types.

Corollary 3.10 Let $L$ be a relational similarity type and $K$ a class of $L$-structures. Then, the following are equivalent.

(i) $K$ is axiomatizable by a set of equality-free sentences.

(ii) (a) $K$ is closed under ultrafilter-products and isomorphic images.

(b) For any L-structure $\mathfrak{A}$, if some ultrafilter-power of $\mathfrak{A}$ lies in $K$ then $\mathfrak{A} \in K$.

(c) For any one-element L-structure $\mathfrak{A}, \mathfrak{A} \in K$ iff there is a $\mathfrak{B} \in \mathbf{H}_{S}^{-1}(\mathfrak{A})$ such that $\mathfrak{B} \in K$ and $|B| \geq 2$.

Proof: That (i) implies conditions (a) and (b) of (ii) is clear. It also implies condition (c), since $K$ is closed under $\mathbf{H}_{S}^{-1}$ and $\mathbf{H}_{S}$. Now assume that (ii) holds. By reasoning as in the proof of Theorem 2.9 but now using an ultrafilter-product instead of an ultraproduct, it is sufficient to show that $\mathfrak{A} \in K$ under the assumption that $\mathfrak{A} \equiv^{-} \mathfrak{B}$ for some $\mathfrak{B} \in K$. If $\mathfrak{A}$ and $\mathfrak{B}$ have at least two elements this follows from Theorem 3.8. If $\mathfrak{A}$ is a one-element structure and $\mathfrak{B}$ is not, $\mathfrak{A} \cong \mathfrak{B}^{*}$. So $\mathfrak{B} \in \mathbf{H}_{S}^{-1}(\mathfrak{A})$. Therefore, by condition (c) of the assumption, we get $\mathfrak{A} \in K$. Now, if $\mathfrak{B}$ is a oneelement structure and $\mathfrak{A}$ is not, there is, by (c), $\mathfrak{C} \in K$ with at least two elements such that $\mathfrak{C} \in \mathbf{H}_{S}^{-1}(\mathfrak{B})$. But then $\mathfrak{A} \equiv^{-} \mathfrak{C}$ and we argue as in the first case. To conclude, note that if both structures are one-element structures, being equality-free equivalent, they must be isomorphic.

The following examples show that Condition (c) in Corollary 3.10 can not be eliminated.

Example 3.11 Let $L=\{P\}$, where $P$ is a monadic relation symbol. Let $K_{1}$ be the class of all one-element $L$-structures and let $K_{2}$ be the class of all $L$-structures having at least two elements. $K_{1}$ and $K_{2}$ are not axiomatizable by a set of equality-free sentences and they satisfy conditions (a) and (b) of Corollary 3.10. $K_{1}$ does not satisfy the implication from left to right in condition (c) and $K_{2}$ does not satisfy the implication from right to left in that condition.

4 Back and forth for equality-free logic The characterization of $\mathfrak{A} \equiv \mathfrak{B}$, for $\mathfrak{A}$ and $\mathfrak{B}$ of the same finite similarity type, in terms of the existence of a winning strategy in an associated game is due to Ehrenfeucht and Fraïssé. Here we obtain an analogous characterization for equality-free logic. The interested reader may find a good exposition of the Ehrenfeucht-Fraïssé theorem in Ebbinghaus, Flum and Thomas [8].

Definition 4.1 Let $\mathfrak{A}$ and $\mathfrak{B}$ be $L$-structures. A relation $p \subseteq A \times B$ is said to be $a$ partial relativeness correspondence iff for any $n$-ary relation symbol $R \in L$ and any $\left\langle a_{1}, b_{1}\right\rangle, \ldots,\left\langle a_{n}, b_{n}\right\rangle \in p$,

$$
\left\langle a_{1}, \ldots, a_{n}\right\rangle \in R^{\mathfrak{A}} \text { iff }\left\langle b_{1}, \ldots, b_{n}\right\rangle \in R^{\mathfrak{B}} .
$$

Definition 4.2 Let $\mathfrak{A}$ and $\mathfrak{B}$ be $L$-structures. $\mathfrak{A}$ and $\mathfrak{B}$ are said to be $n$-finitely relatives via $\left(I_{k}\right)_{k \leq n}$, in symbols $\left(I_{k}\right)_{k \leq n}: \mathfrak{A} \sim_{n} \mathfrak{B}$, iff 
(i) every $I_{k}$ is a nonempty set of partial relativeness correspondences;

(ii) (Forth condition) for any $k+1 \leq n$, any $p \in I_{k+1}$ and any $a \in A$ there is $q \in I_{k}$ such that $q \supseteq p$ and $a \in \operatorname{dom}(q)$;

(iii) (Back condition) for any $k+1 \leq n$, any $p \in I_{k+1}$ and any $b \in B$ there is $q \in I_{k}$ such that $q \supseteq p$ and $b \in \operatorname{rg}(q)$;

(iv) for any $k+1 \leq n$, any $p \in I_{k+1}$, and any constant symbol $c \in L, p \cup$ $\left\{\left\langle c^{\mathfrak{A}}, c^{\mathfrak{B}}\right\rangle\right\} \in I_{k}$

(v) for any $k+1 \leq n$, any $p \in I_{k+1}$, any $m$-ary function symbol $f \in L$ and any $\left\langle a_{1}, b_{1}\right\rangle, \ldots,\left\langle a_{m}, b_{m}\right\rangle \in p$,

$$
p \cup\left\{\left\langle f^{\mathfrak{A}}\left(a_{1}, \ldots, a_{m}\right), f^{\mathfrak{B}}\left(b_{1}, \ldots, b_{m}\right)\right\rangle\right\} \in I_{k} .
$$

We write $\mathfrak{A} \sim_{n} \mathfrak{B}$ when there is $\left(I_{k}\right)_{k \leq n}$ such that $\left(I_{k}\right)_{k \leq n}: \mathfrak{A} \sim_{n} \mathfrak{B}$.

Definition 4.3 For any term $t$ of $L$, let $S(t)$ be the set of subterms of $t$ that are not variables. Given an equality-free formula we define by induction the nested rank of $\varphi$, denoted by $N R(\varphi)$, as follows.

$$
\begin{aligned}
& N R\left(R t_{1} \ldots t_{n}\right)=\left|\bigcup_{1 \leq i \leq n} S\left(t_{i}\right)\right|, \\
& N R(\neg \varphi)=N R(\varphi), \\
& N R(\varphi \vee \psi)=\max \{N R(\varphi), N R(\psi)\}, \\
& N R(\exists x \varphi)=N R(\varphi)+1 .
\end{aligned}
$$

Given $\mathfrak{A}$ and $\mathfrak{B} L$-structures we write

$$
\mathfrak{A} \equiv_{n}^{-} \mathfrak{B}
$$

when $\mathfrak{A}$ and $\mathfrak{B}$ satisfy exactly the same equality-free sentences of nested rank $\leq n$.

Lemma 4.4 For finite similarity types and finite sets $V$ of variables there is up to logical equivalence only a finite number of equality-free formulas in the variables of $V$ and of nested rank $\leq n$.

Proof: $\quad$ Let $L$ be a finite similarity type and $V$ a finite set of variables. Let $\mathcal{T}_{V}^{0}=V$ and for any $n \in \omega$

$$
\mathcal{T}_{V}^{n+1}=\mathcal{T}_{V}^{n} \cup\{c: c \in L\} \cup\left\{f t_{1} \ldots t_{k}: f \in L \text { is } k \text {-adic and } t_{1}, \ldots, t_{k} \in \mathcal{T}_{V}^{n}\right\} .
$$

It is clear that $\mathcal{T}_{V}^{n}$ is finite for every $n \in \omega$. An easy induction on the construction of a term $t$ in the variables of $V$ shows that for any $n \in \omega$, if $|S(t)| \leq n$ then $t \in \mathcal{T}_{V}^{n}$. It follows that for any equality-free atomic formula $R t_{1} \ldots t_{m}$ in the variables of $V$ and any $n \in \omega$, if $N R\left(R t_{1} \ldots t_{m}\right) \leq n$ then $t_{1}, \ldots, t_{m} \in \mathcal{T}_{V}^{n}$. Therefore there is only a finite number of equality-free atomic formulas in the variables of $V$ of nested rank $\leq n$. Using this fact it is easy to finish the proof by induction on the nested rank.

Proposition 4.5 Let $L$ be a finite similarity type and $\mathfrak{A}$ and $\mathfrak{B} L$-structures. Then for any $n \in \omega$,

$$
\mathfrak{A} \equiv_{n}^{-} \mathfrak{B} \text { iff } \mathfrak{A} \sim_{n} \mathfrak{B} .
$$


Proof: $\quad(\Rightarrow)$ Suppose that $\mathfrak{A} \equiv_{n}^{-} \mathfrak{B}$. We define for any $m \leq n, I_{m}$ as the set of all the finite partial relativeness correspondences $p$ such that for any equality-free formula $\varphi\left(y_{1}, \ldots, y_{k}\right)$ with $N R(\varphi) \leq m$ and any $\left\langle a_{1}, b_{1}\right\rangle, \ldots\left\langle a_{k}, b_{k}\right\rangle \in p$,

$$
\mathfrak{A} \models \varphi\left[a_{1}, \ldots, a_{k}\right] \text { iff } \mathfrak{B} \models \varphi\left[b_{1}, \ldots, b_{k}\right] .
$$

Let us see that conditions (i) - (v) of the definition of $\sim_{n}$ hold.

(i) Since $\mathfrak{A} \equiv_{n}^{-} \mathfrak{B}$ we have $\varnothing \in I_{m}$.

(ii) Let $m+1 \leq n, p \in I_{m+1}$ and $p=\left\{\left\langle a_{1}, b_{1}\right\rangle, \ldots,\left\langle a_{k}, b_{k}\right\rangle\right\}$. Suppose $a \in$ $A$. Since $L$ is finite, there is a finite set $X$ of equality-free formulas in the variables $z, y_{1}, \ldots, y_{k}$ and of nested rank $\leq m$, such that any equality-free formula in the variables $z, y_{1}, \ldots, y_{k}$ and of nested rank $\leq m$ is logically equivalent to one formula in this set. Consider now the set $\Phi=\left\{\psi \in X: \mathfrak{A} \models \psi\left[a, a_{1}, \ldots, a_{k}\right]\right\}$. Then $\mathfrak{A}=\exists z \wedge \Phi\left[a_{1}, \ldots, a_{k}\right]$ and since $N R(\exists z \wedge \Phi) \leq m+1$ and $p \in I_{m+1}$, by the assumption $\mathfrak{B} \models \exists z \wedge \Phi\left[b_{1}, \ldots, b_{k}\right]$. Let $b \in B$ be such that $\mathfrak{B} \models \bigwedge \Phi\left[b, b_{1}, \ldots, b_{k}\right]$. Thus, clearly $p \cup\{\langle a, b\rangle\} \in I_{m}$.

(iii) is analogous to (ii).

(iv) is similar to the proof of (v). We prove only this last case.

(v) Let $m+1 \leq n, p \in I_{m+1}$ and $f \in L$ a $k$-ary function symbol. For any equality-free formula $\varphi\left(y_{1}, \ldots, y_{k+1}\right)$ with $N R(\varphi) \leq m$,

$$
\mathfrak{A} \models \varphi\left[a_{1}, \ldots, a_{k}, f^{\mathfrak{A}}\left(a_{1}, \ldots, a_{k}\right)\right] \text { iff } \mathfrak{A} \models \varphi^{\prime}\left[a_{1}, \ldots, a_{k}\right],
$$

where $\varphi^{\prime}$ is obtained by substituting in $\varphi$ the term $f y_{1} \ldots y_{k}$ for the variable $y_{k+1}$. Since $N R\left(\varphi^{\prime}\right) \leq m+1$ and $p \in I_{m+1}$,

$$
\mathfrak{A} \models \varphi^{\prime}\left[a_{1}, \ldots, a_{k}\right] \text { iff } \mathfrak{B} \models \varphi^{\prime}\left[b_{1}, \ldots, b_{k}\right] .
$$

Now

$$
\mathfrak{B} \models \varphi^{\prime}\left[b_{1}, \ldots, b_{k}\right] \text { iff } \mathfrak{B} \models \varphi\left[b_{1}, \ldots, b_{k}, f^{\mathfrak{B}}\left(b_{1}, \ldots, b_{k}\right)\right],
$$

and therefore $p \cup\left\{\left\langle f^{\mathfrak{A}}\left(a_{1}, \ldots, a_{k}\right), f^{\mathfrak{B}}\left(b_{1}, \ldots, b_{k}\right)\right\rangle\right\} \in I_{m}$. Thus we conclude that $\left(I_{m}\right)_{m \leq n}: \mathfrak{A} \sim_{n} \mathfrak{B}$.

$(\Leftarrow)$ Suppose that $\left(I_{m}\right)_{m \leq n}: \mathfrak{A} \sim_{n} \mathfrak{B}$. First we show by induction on $m$ that

(*) If $m \leq n$ and $\varphi=R t_{1} \ldots t_{l}$ is an atomic formula whose free variables are among $x_{1}, \ldots, x_{k}$ and whose nested rank is $\leq m$ then, for any $p \in I_{m}$ and any $\left\langle a_{1}, b_{1}\right\rangle, \ldots,\left\langle a_{k}, b_{k}\right\rangle \in p$ there is $q \in I_{0}$ such that $p \subseteq q$ and for any $i, 1 \leq i \leq l$, $\left\langle t_{i}^{\mathfrak{A}}\left[a_{1}, \ldots, a_{k}\right], t_{i}^{\mathfrak{B}}\left[b_{1}, \ldots, b_{k}\right]\right\rangle \in q$.

The case $m=0$ is clear. Suppose inductively that condition (*) holds for $m$. Let $\varphi=R t_{1} \ldots t_{l}$ with nested rank $\leq m+1 \leq n$, let $p \in I_{m+1}$ and let $\left\langle a_{1}, b_{1}\right\rangle, \ldots,\left\langle a_{k}, b_{k}\right\rangle$ $\in p$. If $N R\left(R t_{1} \ldots t_{l}\right)=0$ we are done. So let $N R\left(R t_{1} \ldots t_{l}\right) \geq 1$. There is a subterm $r=r\left(x_{1}, \ldots, x_{k}\right)$ of $\varphi$ which is either a constant or a term of the form $g x_{i_{1}} \ldots x_{i_{j}}$, where $g$ is a $j$-ary function symbol of $L$ and $i_{1}, \ldots, i_{j} \in\{1, \ldots, k\}$. Let $y$ be a new variable that does not occur in $\varphi$ and for every $i, 1 \leq i \leq l$, let $t_{i}^{\prime}$ be the term obtained from $t_{i}$ by substituting the variable $y$ for the term $r\left(x_{1}, \ldots, x_{k}\right)$. Note that 
$t_{i}^{\prime}=t_{i}^{\prime}\left(x_{1}, \ldots, x_{k}, y\right)$ and that $\varphi^{\prime}=R t_{1}^{\prime} \ldots t_{l}^{\prime}$ has nested rank $\leq m$. By conditions (iv) and (v) of the definition of $\sim_{n}$,

$$
p \cup\left\{\left\langle r^{\mathfrak{A}}\left[a_{1}, \ldots, a_{k}\right], r^{\mathfrak{B}}\left[b_{1}, \ldots, b_{k}\right]\right\rangle\right\} \in I_{m} .
$$

Therefore, by inductive hypothesis, there is $q \in I_{0}$ such that

$$
p \cup\left\{\left\langle r^{\mathfrak{A}}\left[a_{1}, \ldots, a_{k}\right], r^{\mathfrak{B}}\left[b_{1}, \ldots, b_{k}\right]\right\rangle\right\} \subseteq q
$$

and for any $i, 1 \leq i \leq l$,

$$
\left\langle t_{i}^{\prime \mathfrak{A}}\left[a_{1}, \ldots, a_{k}, r^{\mathfrak{A}}\left[a_{1}, \ldots, a_{k}\right]\right], t_{i}^{\prime \mathfrak{B}}\left[b_{1}, \ldots, b_{k}, r^{\mathfrak{B}}\left[b_{1}, \ldots, b_{k}\right]\right]\right\rangle \in q .
$$

Then, clearly $p \subseteq q$ and for any $i, 1 \leq i \leq l,\left\langle t_{i}^{\mathfrak{A}}\left[a_{1}, \ldots, a_{k}\right], t_{i}^{\mathfrak{B}}\left[b_{1}, \ldots, b_{k}\right]\right\rangle \in q$. Therefore, Condition $(*)$ holds.

Let $m \leq n$. We prove now by induction on $\varphi$ that for any equality-free formula $\varphi\left(y_{1}, \ldots, y_{k}\right)$ with nested rank $\leq m$, any $p \in I_{m}$ and any $\left\langle a_{1}, b_{1}\right\rangle, \ldots,\left\langle a_{k}, b_{k}\right\rangle \in p$,

$$
\mathfrak{A} \models \varphi\left[a_{1}, \ldots, a_{k}\right] \text { iff } \mathfrak{B} \models \varphi\left[b_{1}, \ldots, b_{k}\right] .
$$

If $\varphi$ is atomic this is clear by Condition $(*)$. The cases $\neg$ and $\vee$ are immediate. Let $\varphi=\exists y \psi$ and suppose inductively that condition 11 holds for $\psi$. If $\mathfrak{A} \models$ $\exists y \psi\left[a_{1}, \ldots, a_{k}\right]$ then there is $a \in A$ such that $\mathfrak{A} \models \psi\left[a, a_{1}, \ldots, a_{k}\right]$. Observe that $m \geq N R(\exists y \psi) \geq 1$. Now $N R(\psi) \leq m-1$. Hence by (ii) of the definition of $\sim_{n}$ there is $q \in I_{m-1}$ such that $q \supseteq p$ and $a \in \operatorname{dom}(q)$. Let $b \in B$ be such that $\langle a, b\rangle \in q$. By inductive hypothesis, $\mathfrak{B} \models \psi\left[b, b_{1}, \ldots, b_{k}\right]$ and therefore $\mathfrak{B} \models \exists y \psi\left[b_{1}, \ldots, b_{k}\right]$. The other direction is proved analogously using condition (iii) of the definition of $\sim_{n}$. By Condition 11 we conclude that $\mathfrak{A} \equiv_{n}^{-} \mathfrak{B}$.

Observe that in the previous proof when proving that $\mathfrak{A} \sim_{n} \mathfrak{B}$ implies $\mathfrak{A} \equiv_{n}^{-} \mathfrak{B}$ we do not make any use of the fact that $L$ is a finite similarity type.

Theorem 4.6 Let L be a finite similarity type and $\mathfrak{A}$ and $\mathfrak{B}$ L-structures, then

$$
\mathfrak{A} \equiv^{-} \mathfrak{B} \text { iff } \mathfrak{A} \sim_{n} \mathfrak{B}, \text { for any } n \in \omega .
$$

\section{Proof: By Proposition 4.5 .}

There is also an infinite back and forth for equality-free logic. We omit the proofs and limit ourselves to stating the basic facts. The reader may find it useful to consult Barwise $[1$ or Ebbinghaus et al. 8 .

Definition 4.7 Let $\mathfrak{A}$ and $\mathfrak{B}$ be $L$-structures. $\mathfrak{A}$ and $\mathfrak{B}$ are said to be partially relatives via $I$ (written $I: \mathfrak{A} \sim{ }_{p} \mathfrak{B}$ ) iff

(i) $I$ is a nonempty set of partial relativeness correspondences;

(ii) For any $p \in I$ and any $a \in A$ there is $q \in I$ such that $q \supseteq p$ and $a \in \operatorname{dom}(q)$;

(iii) For any $p \in I$ and any $b \in B$ there is $q \in I$ such that $q \supseteq p$ and $b \in \operatorname{rg}(q)$;

(iv) For any $p \in I$ and any constant symbol $c \in L, p \cup\left\{\left\langle c^{\mathfrak{A}}, c^{\mathfrak{B}}\right\rangle\right\} \in I$; 
(v) For any $p \in I$, any $k$-ary function symbol $f \in L$ and any $\left\langle a_{1}, b_{1}\right\rangle, \ldots,\left\langle a_{k}, b_{k}\right\rangle$ $\in p$,

$$
p \cup\left\{\left\langle f^{\mathfrak{A}}\left(a_{1}, \ldots, a_{k}\right), f^{\mathfrak{B}}\left(b_{1}, \ldots, b_{k}\right)\right\rangle\right\} \in I .
$$

And we write $\mathfrak{A} \sim_{p} \mathfrak{B}$ when there is $I$ such that $I: \mathfrak{A} \sim{ }_{p} \mathfrak{B}$.

It is easy to see that for any $\mathfrak{A}$ and $\mathfrak{B}$

$$
\mathfrak{A} \sim_{p} \mathfrak{B} \text { iff } \mathfrak{A} \equiv{ }_{\infty \omega}^{-} \mathfrak{B}
$$

where $\mathfrak{A} \equiv_{\infty \omega}^{-} \mathfrak{B}$ means that $\mathfrak{A}$ and $\mathfrak{B}$ satisfy the same equality-free sentences of $L_{\infty \omega}$. It is clear that $\mathfrak{A} \sim \mathfrak{B}$ implies $\mathfrak{A} \sim_{p} \mathfrak{B}$ and that $\mathfrak{A} \sim_{p} \mathfrak{B}$ implies $\mathfrak{A} \sim_{n} \mathfrak{B}$ for each $n \in \omega$. Moreover, for countable structures $\mathfrak{A}$ and $\mathfrak{B}$,

$$
\mathfrak{A} \sim \mathfrak{B} \text { iff } \mathfrak{A} \sim{ }_{p} \mathfrak{B} .
$$

There is a notion of $\omega$-saturation for equality-free logic. We say that a structure $\mathfrak{A}$ is $\omega$-equality-free saturated if for every finite $X \subseteq A$, every consistent set $\Sigma(x)$ of equality-free formulas of $L(X)$ is realized in $\mathfrak{A}$, that is, there is $a \in A$ such that for every $\varphi(x) \in \Sigma(x), \mathfrak{A}_{X} \models \varphi[a]$. Obviously every structure has an $L^{-}$-extension which is $\omega$-equality-free saturated. By standard arguments we get that for $\omega$-equality-free saturated structures $\mathfrak{A}$ and $\mathfrak{B}$

$$
\mathfrak{A} \sim_{p} \mathfrak{B} \text { iff } \mathfrak{A} \equiv{ }^{-} \mathfrak{B} .
$$

Hence, as in the case of first-order logic with equality, infinite back and forth is a useful tool to prove completeness of theories: $T$ is a complete theory in equality-free logic iff for any $\omega$-equality-free saturated models $\mathfrak{A}$ and $\mathfrak{B}$ of $T$ we have $\mathfrak{A} \sim_{p} \mathfrak{B}$.

Acknowledgments The first two authors were partially supported by grant PB94-0854 of DGYCIT and the third one by grant PB94-0920 of DGYCIT.

\section{REFERENCES}

[1] Barwise, J., "Back and forth through infinitary logic," pp. 5-34 in Studies in Model Theory, edited by M. Morley, Studies in Mathematics 8, Mathematical Association of America, Washington, D.C., 1973.MR 49:7116 4

[2] Bloom, S. L., "Some Theorems on Structural Consequence Operations," Studia Logica, vol. 34 (1975), pp. 1-9.Zbl 0311.02016|MR 53:2675, 1

[3] Blok, W., and D. Pigozzi, "Protoalgebraic Logics," Studia Logica, vol. 45 (1986), pp. 337-369. Zbl 0622.03020 MR 88e:03045 1.2.2

[4] Blok, W., and D. Pigozzi, “Algebraizable logics," Memoirs of the American Mathematical Society, vol. 1 (1989), p. 396. Zbl 0664.03042 MR 90d:03140 1

[5] Blok, W., and D. Pigozzi, "Algebraic Semantics for Universal Horn Logic without Equality," in Universal Algebra and Quasigroups, edited by A. Romanowska and J. D. H. Smith, Heldermann Verlag, Berlin, 1992.Zbl 0768.03008 MR 94g:03021]1.3

[6] Czelakowski, J., "Equivalential Logics I,” Studia Logica, vol. 40 (1981), pp. 227-236. Zbl 0476.03032|MR 84k:03072 2 
[7] Chang, C. C., and H. J. Keisler, Model Theory, North Holland, Amsterdam 1973. Zbl 0697.03022 |MR 91c:03026 2.12.13

[8] Ebbinghaus, H. D., J. Flum, and W. Thomas, Mathematical Logic, Springer-Verlag, Berlin 1984.Zbl 0556.03001||MR 85a:030014.4.

[9] Monk, J. D., Mathematical Logic, Springer-Verlag, New York, 1976.Zbl 0354.02002 MR 57:5656 1,1,12,3

[10] Shelah, S., "Every two elementarily equivalent models have isomorphic ultrapowers," Israel Journal of Mathematics, vol. 10 (1971), pp. 224-233.Zbl 0224.02045 MR 45:6608 3

Departament de Lògica, Història i Filosofia de la Ciència

Universitat de Barcelona

email: casanova@cerber.mat.ub.es

email: jansana@cerber.mat.ub.es

Àrea de Lògica i Filosofia de la Ciència

Universitat Autònoma de Barcelona

email:dellunde@cc.uab.es 